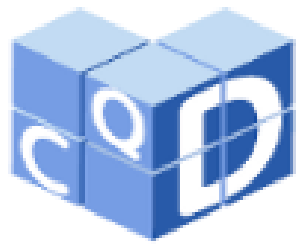

Revista Eletrônica Paulista de Matemática

ISSN 2316-9664

Volume 16, dez. 2019

Rodrigo José Martinelli Biglia Andrade

UNIMEP - Universidade Metodista de Piracicaba

E-mail: contato@pontoabc.com

\section{Resolução de uma equação do quinto grau.}

Resolution of an equation of the fifth degree.

\section{Resumo}

De acordo com o Teorema de Abel-Ruffini, uma equação de quinto grau, quando completa, ou seja, dados todos os seus coeficientes, como por exemplo, $x^{5}+2 x^{4}+5 x^{3}+10 x^{2}+x+10=0$, não pode ser resolvida por radicais. Devido a esse teorema, não existe até então uma fórmula ou técnica para resolver todas as equações de quinto grau por meio de radicais. Assim, verificaremos nesse artigo, uma maneira de resolver casos específicos de equações do quinto grau com o uso de uma transformação, que é a substituição da variável $x$ de uma equação de quinto grau, por $\frac{(-b+t)}{5 a}$, para que seja possível eliminar o termo de quarto grau da equação, e com o uso de um polinômio de décimo grau faremos uma mudança de variável para conseguirmos resolver uma equação de quinto grau que tenha somente soluções inteiras, como é o caso de: $x^{5}+112 x^{4}+4428 x^{3}+76032 x^{2}+551232 x+1244160=0$.

Palavras-chave: Equação do quinto grau, Raízes, Transformação de Martinelli, Igualdade de Martinelli.

\begin{abstract}
According to Abel-Ruffini's theorem, a fifth-degree equation, when complete, that is, given all its coefficients, for example, $x^{5}+2 x^{4}+5 x^{3}+10 x^{2}+x+10=0$, it is impossible be solved by radicals. Due to this theorem, there is as yet no formula or technique for solving all fifth degree equations by radicals. Thus, we will examine in this paper a way to solve specific cases of fifth degree equations using a transformation, which is the substitution of the variable $\mathrm{x}$ of a fifth degree equation by $\frac{(-b+t)}{5 a}$, for eliminate the fourth degree term from the equation, and using a tenth degree polynomial we will make a variable change to solve a fifth degree equation that has only integer solutions, as the case of: $x^{5}+112 x^{4}+4428 x^{3}+76032 x^{2}+551232 x+1244160=0$.
\end{abstract}

Keywords: Fifth degree equation, Roots, Transformation of Martinelli, Equality of Martinelli. 


\section{Introdução}

Muitos acreditam que é impossível resolver uma equação de quinto grau completa pelo fato de muitas tentativas terem falhado no que se refere ao desenvolvimento de uma fórmula para esse fim. Mas, as equações triviais como $\mathrm{x}^{5}-32=0$ são solúveis.

No entanto, com a técnica que iremos ver nesse artigo, poderemos resolver casos específicos de equação do quinto grau de maneira analítica.

A técnica para solucionar equações possíveis de serem resolvidas consiste em criar uma equação de décimo grau, que é a igualdade de Martinelli, onde nela conterá pelo menos uma solução (dependendo dos coeficientes da equação do quinto grau) racional, a qual poderá ser escolhida para encontrar as raízes da equação já transformada. No entanto, para as equações de quinto grau que envolvem somente raízes irracionais ou complexas. Essa técnica também funciona em alguns casos específicos como $x^{5}-1121 x-2080=0$ onde podemos separar a equação em duas de grau menor.

Como iremos resolver a equação $x^{5}+112 x^{4}+4428 x^{3}+76032 x^{2}+551232 x+1244160=0$ seguiremos a ideia de tornar as raízes inteiras em múltiplas de 5 (cinco). Para isso usaremos a transformação de Martinelli. Após fazer isso bastará dividir a equação de décimo grau (que será explicada e provada matematicamente a posteriori) pela equação de quinto grau transformada. Assim, no resto da divisão, em muitos casos, surgirá uma equação de quarto grau resolvível (pelo método de Descartes, por exemplo). Mas, em outros casos, o resto poderá ser 0 (zero) e isso mostra que a equação foi separada em duas de quinto grau. Para corrigir isso, basta fazer novamente uma mudança de variável para obter outras soluções que serão múltiplas de 5 (cinco) e surgirá um resto diferente de 0 . No entanto, não veremos como funciona o método de Descartes ou outro método. $\mathrm{O}$ foco do artigo é mostrar uma maneira, técnica, de encontrar pelo menos uma solução de uma equação de quinto grau quando suas soluções forem inteiras.

A ideia é separar a equação de quinto grau em duas de grau menores como uma de terceiro grau e uma de segundo grau. Para fazer isso podemos considerar o seguinte:

$$
\left(\mathrm{x}^{3}+\mathrm{kx} \mathrm{x}^{2}+\mathrm{kx}+\mathrm{m}\right)\left(\mathrm{x}^{2}-\mathrm{kx}+\mathrm{n}\right)=0,
$$

$\mathrm{e}$

$$
\left(\mathrm{x}^{3}+k x^{2}+\mathrm{lx}+\mathrm{m}\right)\left(\mathrm{x}^{2}-\mathrm{kx}+\mathrm{n}\right)=0 .
$$

Os coeficientes $\mathrm{k}$, $\mathrm{n}$ e $\mathrm{m}$ são, na maioria das vezes, racionais, sendo $\mathrm{k}$ o coeficiente comum entre as duas igualdades (que será um coeficiente de quarto grau suprimido se expandirmos uma dessas igualdades) e m e $\mathrm{n}$ as duas incógnitas independentes.

Na equação (1) nota-se que temos $\left(\mathrm{x}^{3}+\mathrm{kx}^{2}+\mathrm{kx}+\mathrm{m}\right)$ que multiplica $\left(\mathrm{x}^{2}-\mathrm{kx}+\mathrm{n}\right)$. Se tomarmos por exemplo, $\mathrm{k}=10$ e substituirmos valores nas incógnitas $\mathrm{m}$ e $\mathrm{n}$, poderemos resolver essa equação sem problema algum por radicais. E, se expandirmos a equação (1) antes, notaremos que se trata de uma equação do quinto grau solúvel por radicais, mas, o mesmo não ocorre se colocarmos valores arbitrários nos coeficientes de uma equação de quinto grau como por exemplo, $x^{5}+x+3=0$. 
O método aqui apresentado para a resolução de casos específicos de equação de quinto grau, pelo fato de ser mais didático, poderá abranger as salas de aula de todo o país por ser mais simples de identificar suas raízes. O contrário ocorre no artigo GREEN; 1978 que mostra uma solução possível, mas mais complexa, com simbologias difíceis de serem memorizadas por um estudante iniciante na matemática por exemplo, e isso torna o estudo oneroso e maçante.

O objetivo do presente artigo é utilizar uma linguagem de fácil compreensão e uma leitura convidativa ao leitor para que o mesmo saiba como utilizar essa técnica de resolver uma equação do quinto grau quando se deparar com uma equação possível de ser solucionada por radicais do modo não trivial conforme já comentado.

\section{Sobre o teorema de Abel-Ruffini e a teoria de Galois.}

Algumas ideias acerca da teoria de Galois e do Teorema de Abel-Ruffini.

O teorema afirma que: "Não existe fórmula ou técnica para solucionar todas as equações de quinto grau. Mas, o teorema não afirma que é impossível solucionar todas as equações de quinto grau, algumas podem ser resolvidas por radicais...” (ZOLADEK, 2000).

Já a teoria de Galois afirma que: "No nível mais básico, ela usa grupo de permutações para descrever como as várias raízes de uma certa equação polinomial estão relacionadas umas com as outras..." (ARTIN, 1998).

Então, de acordo com os seus coeficientes, há solução para alguns casos. E é nesse aspecto que trataremos de apresentar uma técnica capaz de solucionar um caso específico de equação do quinto grau de maneira analítica.

\subsection{O primeiro passo: A transformação de Martinelli}

Para se chegar na transformação de Martinelli é preciso desenvolver a fórmula que resolve uma equação de quinto grau por iterações. Vejamos:

$$
a x^{5}+b x^{4}+c x^{3}+d x^{2}+e x+f=0 .
$$

Agora, desenvolvendo a equação (3) para que consigamos um binômio de quinto grau, segue que:

$$
a x^{5}+b x^{4}=-c x^{3}-d x^{2}-e x-f
$$

Devemos agora multiplicar o lado esquerdo e direito da equação (4) por $3125 \mathrm{a}^{4}$ com o objetivo de criar, do lado esquerdo da equação, um binômio elevado á quinta potência.

$$
3125 a^{5} x^{5}+3125 a^{4} b x^{4}=-3125 a^{4} c x^{3}-3125 a^{4} d x^{2}-3125 a^{4} e x-3125 a^{4} f \text {. }
$$

Agora, em ambos os membros da equação (5), adicione:

$$
1250 a^{3} b^{2} x^{3}+250 a^{2} b^{3} x^{2}+25 a b^{4} x+b^{5}
$$


Assim, teremos:

$(5 a x+b)^{5}=1250 a^{3} b^{2} x^{3}+250 a^{2} b^{3} x^{2}+25 a b^{4} x+b^{5}-3125 a^{4} c x^{3}-3125 a^{4} d x^{2}-3125 a^{4} e x-3125 a^{4} f$

Isolando $\mathrm{x}$ no termo $(5 \mathrm{ax}+\mathrm{b})^{5}$, teremos a expressão:

$$
x=\frac{-b+\sqrt[5]{1250 a^{3} b^{2} x^{3}+250 a^{2} b^{3} x^{2}+25 a b^{4} x+b^{5}-3125 a^{4} c x^{3}-3125 a^{4} d x^{2}-3125 a^{4} e x-3125 a^{4} f}}{5 a} .
$$

Colocando os termos $\mathrm{x}^{3}, \mathrm{x}^{2} \mathrm{e} \mathrm{x}$ em evidência teremos:

$$
x=\frac{-b+\sqrt[5]{b^{5}+125 a^{3}\left(10 b^{2}-25 a c\right) x^{3}+125 a^{2}\left(2 b^{3}-25 a^{2} d\right) x^{2}+25 a\left(b^{4}-125 a^{3} e\right) x-3125 a^{4} f}}{5 a} .
$$

Agora iremos substituir x na equação (7) pela seguinte fração $\frac{(-b+t)}{5 a}$ e expandindo os termos chegaremos na transformação:

$\mathrm{t}^{5}$

$-\left(10 b^{2}-25 a c\right) t^{3}$

$-\left(-3 b\left(10 b^{2}-25 a c\right)+5\left(2 b^{3}-25 a^{2} d\right)\right) t^{2}$

$-\left(3 b^{2}\left(10 b^{2}-25 a c\right)-10 b\left(2 b^{3}-25 a^{2} d\right)+5\left(b^{4}-125 a^{3} e\right)\right) t$

$-\left(-b^{3}\left(10 b^{2}-25 a c\right)+5 b^{2}\left(2 b^{3}-25 a^{2} d\right)-5 b\left(b^{4}-125 a^{3} e\right)+b^{5}-3125 a^{4} f\right)=0$.

Essa é então a transformação que iremos usar para poder resolver uma equação do quinto grau específica.

Depois da transformação usaremos o termo $\frac{-\mathrm{b}+\mathrm{t}}{5 \mathrm{a}}$ para descobrir uma solução (raiz) da equação de quinto grau original. Como iremos fazer uma mudança de variável para poder deixar as raízes múltiplas de 5, no caso da equação de quinto grau transformada e na equação de décimo grau, que virá com o desenvolvimento da igualdade de Martinelli, devemos adicionar ao t esse número inteiro para obter a solução. Veremos isso com mais detalhes ao decorrer do artigo com um exemplo.

O segredo então para se obter as soluções de uma equação de quinto grau possível é separá-la em duas de grau menores como em (1) e (2).

Se expandirmos a equação (1) temos:

$$
x^{5}+x^{3} n-k^{2} x^{3}+k x^{2} n+k x^{3}-k^{2} x^{2}+k x n+m x^{2}-k x m+m n=0 .
$$

Agora expandindo a equação (2), temos:

$$
x^{5}+n x^{3}-k^{2} x^{3}+k x^{2} n+l x^{3}-k l x^{2}+\ln x+m x^{2}-k m x+m n=0 .
$$


Veja que não há o termo de quarto grau nas equações (9) e (10). Então toda equação de quinto grau com o termo de quarto grau (a equação original no caso), pode ser transformada para uma outra sem o coeficiente de quarto grau.

Feito isso, vamos colocar em uma tabela os coeficientes das equações (9) e (10), cada coeficiente, em seu respectivo lugar.

Coeficientes da equação (9)

\begin{tabular}{|c|c|c|c|}
\hline $\mathrm{x}^{3}$ & $\mathrm{x}^{2}$ & $\mathrm{x}$ & Termo independente \\
\hline $\mathrm{k}+\mathrm{n}-\mathrm{k}^{2}=\mathrm{C}_{2}$ & $-\mathrm{k}^{2}+\mathrm{kn}+\mathrm{m}=\mathrm{D}_{2}$ & $\mathrm{kn}-\mathrm{km}=\mathrm{E}_{2}$ & $\mathrm{mn}=\mathrm{F}$ \\
\hline
\end{tabular}

Coeficientes da equação (10)

\begin{tabular}{|c|c|c|c|}
\hline $\mathrm{x}^{3}$ & $\mathrm{x}^{2}$ & $\mathrm{x}$ & Termo independente \\
\hline $\mathrm{l}+\mathrm{n}-\mathrm{k}^{2}=\mathrm{C}$ & $\mathrm{nk}-\mathrm{k}+\mathrm{m}=\mathrm{D}$ & $\mathrm{nl}-\mathrm{km}=\mathrm{E}$ & $\mathrm{mn}=\mathrm{F}$ \\
\hline
\end{tabular}

\subsection{O segundo passo: A igualdade de Martinelli}

Tomemos como exemplo a seguinte equação:

$$
x^{2}+3 x+2=0
$$

Se substituirmos x pela soma das raízes da equação (11) teremos $2=0$, que seria um absurdo, mas, com essa ideia, podemos criar uma equação de segundo grau utilizando os termos $\mathrm{C}, \mathrm{C}_{2}, \mathrm{D}, \mathrm{D}_{2}$, E e $\mathrm{E}_{2}$ da seção 2.1 como mostrado na tabela anterior. Igualando a equação que será criada com $\left(-\mathrm{k}^{2}+\mathrm{n}+\mathrm{k}-\mathrm{C}\right) \mathrm{n}$ teremos, do lado direito da equação, a mesma coisa que $\mathrm{E}_{2}-\mathrm{E}$, pois $E_{2}$ é o mesmo que $k n-k m$ e $-E$ é o mesmo que $-E=-n l+k m$. A soma de $E_{2}-E$ resultará em kn-nl sendo $l=C-n+k^{2}$. Assim temos $k n-n\left(C-n+k^{2}\right)$ que é o mesmo que $\left(-k^{2}+n+k-C\right) n$.

Para criarmos uma equação de segundo grau onde uma das soluções da equação será a soma de duas soluções de uma equação de quinto grau após ter passado pela transformação (8), devemos seguir essa lógica: Temos a forma geral da equação de segundo grau que é $a x^{2}+b x+c=0$, os coeficientes da equação de segundo grau que será criada serão $a=C_{2}-C$, $\mathrm{b}=\mathrm{D}_{2}-\mathrm{D}, \quad \mathrm{c}=\mathrm{E}_{2}-\mathrm{E}$ e no lado direito da equação temos que adicionar ($\left.\mathrm{k}^{2}+\mathrm{n}+\mathrm{k}-\mathrm{C}\right) \mathrm{n}$ pois do lado esquerdo vai restar $\mathrm{E}_{2}-\mathrm{E}$.

Observe que a equação de décimo grau que virá com o desenvolvimento dessa lógica conterá raízes provenientes da soma de duas das raízes da equação de quinto grau. Ou seja, estaremos expandindo a equação de quinto grau. Por exemplo, se as raízes da equação de quinto grau, após passar pela transformação (8), forem $5,15,20,25,30$, então as raízes da equação de décimo grau serão 20,25,30,35,35,40,45,45,50,55.

Essa ideia vai nos dar a possibilidade de isolar a incógnita $n$ da equação (2). $\mathrm{O}$ objetivo é formar uma equação de décimo grau com a incógnita k. Como $\mathrm{a}=\mathrm{C}_{2}-\mathrm{C}$ e $\mathrm{b}=$ $\mathrm{D}_{2}-\mathrm{D}$ de acordo com a tabela da seção anterior, segue que $\mathrm{a}=\mathrm{k}+\mathrm{n}-\mathrm{k}^{2}-\mathrm{C}, \mathrm{b}=-$ $\mathrm{k}^{2}+\mathrm{kn}+\mathrm{m}-\mathrm{D}, \mathrm{c}=\mathrm{nk}-\mathrm{km}-\mathrm{E}$ e o membro direito da equação será $\left(-\mathrm{k}^{2}+\mathrm{n}+\mathrm{k}-\mathrm{C}\right) \mathrm{n}$. Desse modo, fazendo as substituições em $\mathrm{ak}^{2}+\mathrm{bk}+\mathrm{c}=0$ temos: 


$$
\begin{aligned}
\left(-\mathrm{k}^{2}+\mathrm{n}+\mathrm{k}-\mathrm{C}\right) \mathrm{k}^{2}+\left(-\mathrm{k}^{2}+\mathrm{nk}+\mathrm{m}-\mathrm{D}\right) \mathrm{k}+\mathrm{nk}-\mathrm{km}-\mathrm{E} & =\left(-\mathrm{k}^{2}+\mathrm{n}+\mathrm{k}-\mathrm{C}\right) \mathrm{n} . \\
\left(-\mathrm{k}^{4}+\mathrm{nk}^{2}+\mathrm{k}^{3}-\mathrm{Ck}^{2}\right)+\left(-\mathrm{k}^{3}+\mathrm{nk}^{2}+\mathrm{km}-\mathrm{Dk}\right)+\mathrm{nk}-\mathrm{km}-\mathrm{E} & =\left(-\mathrm{k}^{2}+\mathrm{n}+\mathrm{k}-\mathrm{C}\right) \mathrm{n} . \\
-\mathrm{k}^{4}+2 \mathrm{nk}^{2}-\mathrm{Ck}^{2}-\mathrm{Dk}+\mathrm{nk}-\mathrm{E} & =\left(-\mathrm{k}^{2}+\mathrm{n}+\mathrm{k}-\mathrm{C}\right) \mathrm{n} . \\
-\mathrm{k}^{4}+2 \mathrm{nk}^{2}-\mathrm{Ck}^{2}-\mathrm{Dk}+\mathrm{nk}-\mathrm{E} & =-\mathrm{nk}^{2}+\mathrm{n}^{2}+\mathrm{kn}-\mathrm{Cn} . \\
-\mathrm{k}^{4}-\mathrm{Ck}^{2}-\mathrm{Dk}-\mathrm{E} & =-3 \mathrm{nk}^{2}+\mathrm{n}^{2}-\mathrm{Cn} .
\end{aligned}
$$

Isolando n, temos:

$$
\mathrm{n}=\frac{\mathrm{k}^{4}+\mathrm{Ck}^{2}+\mathrm{Dk}+\mathrm{E}}{3 \mathrm{k}^{2}-\mathrm{n}+\mathrm{C}}
$$

Com isso, podemos agora usar mais uma manipulação algébrica e obter mais uma igualdade. Lembrando que $\mathrm{D}=\mathrm{nk}-\mathrm{lk}+\mathrm{m}, \mathrm{l}=\mathrm{k}^{2}-\mathrm{n}+\mathrm{C}$ e $\mathrm{F}=\mathrm{mn}$, reescrevemos $\mathrm{D}$ como:

$$
n k-\left(k^{2}-n+C\right) k+\frac{F}{n}=D \text {. }
$$

Isolando $\mathrm{n}^{2}$ em (13):

$$
\mathrm{n}^{2}=\frac{\mathrm{nk}^{3}+\mathrm{nCk}-\mathrm{F}+\mathrm{Dn}}{2 \mathrm{k}}
$$

Temos duas igualdades importantes: a equação (12) e a equação (14) que terão como objetivo auxiliar na criação de um polinômio de décimo grau onde k será a incógnita.

Com o uso da equação (13) podemos definitivamente isolar a incógnita n que está na equação (12), isto é:

$$
\mathrm{n}=\frac{2\left(\mathrm{k}^{5}+\mathrm{Ck}^{3}+\mathrm{Dk}{ }^{2}+\mathrm{Ek}\right)-\mathrm{F}}{5 \mathrm{k}^{3}+\mathrm{Ck}-\mathrm{D}}
$$

Podemos substituir n e obter uma equação que culminará numa equação de décimo grau. Após a manipulação das raízes com uma troca de variável será possível dividir a equação de décimo grau com a equação de quinto grau onde, na maioria das vezes, resultará em um polinômio de quarto grau como resto. Uma de suas soluções será também a solução da equação de quinto grau após ser transformada.

Na igualdade (12) podemos substituir n que está no denominador pela equação (14). Assim teremos uma nova equação.

$$
\begin{gathered}
\frac{\mathrm{k}^{4}+\mathrm{Ck}^{2}+\mathrm{Dk}+\mathrm{E}}{3 \mathrm{k}^{2}-\frac{2\left(\mathrm{k}^{5}+\mathrm{Ck}^{3}+\mathrm{Dk} \mathrm{k}^{2}+\mathrm{Ek}\right)-\mathrm{F}}{5 \mathrm{k}^{3}+\mathrm{Ck}-\mathrm{D}}=\frac{2\left(\mathrm{k}^{5}+\mathrm{Ck}^{3}+\mathrm{Dk}^{2}+\mathrm{Ek}\right)-\mathrm{F}}{5 \mathrm{k}^{3}+\mathrm{Ck}-\mathrm{D}} .} \\
\frac{\left(\mathrm{k}^{4}+\mathrm{Ck}^{2}+\mathrm{Dk}+\mathrm{E}\right)\left(5 \mathrm{k}^{3}+\mathrm{Ck}-\mathrm{D}\right)}{13 \mathrm{k}^{5}+6 \mathrm{Ck}^{3}-5 \mathrm{Dk}^{2}+\left(-2 \mathrm{E}+\mathrm{C}^{2}\right) \mathrm{k}+\mathrm{F}-\mathrm{DC}}=\frac{2\left(\mathrm{k}^{5}+\mathrm{Ck}^{3}+\mathrm{Dk}^{2}+\mathrm{Ek}\right)-\mathrm{F}}{5 \mathrm{k}^{3}+\mathrm{Ck}-\mathrm{D}} .
\end{gathered}
$$


A equação (16) é o mesmo que uma equação polinomial de décimo grau e é esta a igualdade de Martinelli:

$$
\begin{aligned}
& \left(\mathrm{k}^{4}+\mathrm{Ck}^{2}+\mathrm{Dk}+\mathrm{E}\right)\left(5 \mathrm{k}^{3}+\mathrm{Ck}+\mathrm{D}\right)^{2}- \\
& \left(2\left(\mathrm{k}^{5}+\mathrm{Ck}^{3}+\mathrm{Dk}{ }^{2}+\mathrm{Ek}\right)-\mathrm{F}\right)\left(13 \mathrm{k}^{5}+6 \mathrm{Ck}^{3}-5 \mathrm{Dk}^{2}+\left(-2 \mathrm{E}+\mathrm{C}^{2}\right) \mathrm{k}+\mathrm{F}-\mathrm{DC}\right)=0,
\end{aligned}
$$

em que k representa as soluções da equação (17) e as letras C, D, E e F são os coeficientes da equação transformada (8).

Cada solução da equação (17) será a soma de duas raízes de um polinômio, por exemplo o polinômio possível de ser resolvido que está no resumo deste artigo.

O motivo de escolhermos a equação (12) para substituirmos a incógnita n dada na equação (15) é pelo fato de ser mais simples a manipulação algébrica que resultará no polinômio (17).

\section{A solução de uma equação de quinto grau}

Após obtermos a igualdade (17) podemos usar uma mudança de variável que consiste em fazer com que todas as raízes de um polinômio fiquem múltiplas de 5. Vejamos um exemplo:

Digamos que todas as raízes são inteiras, assim, o último termo, o termo independente no caso, pode assumir qualquer valor como, por exemplo, -1879146. É lógico que esse número não é múltiplo de 5, pois se dividirmos esse número por 5 teremos o resultado -375829,2. Mas, se acrescentarmos 1 ou se somarmos -4 teremos um múltiplo de 5.

É claro que não teremos exatamente esse número quando mudarmos uma variável. Vejamos como ficaria um exemplo:

$$
\mathrm{k}^{5}-3140 \mathrm{k}^{3}+18990 \mathrm{k}^{2}+2290435 \mathrm{k}-30957678=0
$$

Utilizando uma mudança de variável teremos:

$$
(\mathrm{k}+3)^{5}-3140(\mathrm{k}+3)^{3}+18990(\mathrm{k}+3)^{2}+2290435(\mathrm{k}+3)-30957678=0,
$$

ou seja,

$$
\mathrm{k}^{5}+15 \mathrm{k}^{4}-3050 \mathrm{k}^{3}-9000 \mathrm{k}^{2}+2320000 \mathrm{k}-24000000=0 .
$$

Veja que o último termo é múltiplo de 5.

Com o uso da equação de décimo grau, teremos algumas soluções da equação de quinto grau transformada (terá, em muitos casos, apenas uma solução ou ela irá separar a equação de décimo grau em duas de quinto grau). Sem nenhum problema podemos dividir essa equação de décimo grau pela equação de quinto grau transformada e isso garante, no resto da divisão, um polinômio de grau inferior ou igual a 4 para podermos saber uma das soluções da equação de quinto grau original. 
Após resolver a equação, que aparece no resto da divisão do polinômio de décimo grau, podemos inserir na equação de quinto grau transformada as raízes desse polinômio e verificar qual delas são soluções da equação de quinto grau transformada.

Vejamos agora um exemplo (que está no resumo desse artigo) de uma equação de quinto grau com soluções inteiras:

$$
x^{5}+112 x^{4}+4428 x^{3}+76032 x^{2}+551232 x+1244160=0
$$

Queremos saber as soluções da equação (18). Passando pela transformação (8) teremos o seguinte:

$$
t^{5}-14740 t^{3}+407360 t^{2}+21207360 t-511926272=0 .
$$

Agora queremos saber uma das soluções da equação (18) para poder encontrar uma das soluções da equação (17).

Temos que o coeficiente de $t^{5}$ é $A=1$, o coeficiente de $t^{4}$ é $B=0$, o coeficiente de $t^{3}$ é $\mathrm{C}=-14740$, o coeficiente de $\mathrm{t}^{2}$ é $\mathrm{D}=407360$, o coeficiente de $\mathrm{t}$ é $\mathrm{E}=21207360$ e por fím, o termo independente é $\mathrm{F}=-51192672$.

Agora iremos substituir os valores de A,B,C,D,E,F na equação (17) que resulta em uma equação de décimo grau. Perceba que queremos encontrar as raízes k que, por sua vez, terá algum $\mathrm{k}$ igual a $\mathrm{t}$ após a mudança de variável.

Fazendo tal substituição, teremos:

$$
\begin{aligned}
& \left(\mathrm{k}^{4}-14740 \mathrm{k}^{2}+407360 \mathrm{k}+21207360\right)\left(5 \mathrm{k}^{3}-14740 \mathrm{k}-407360\right)^{2}-\left(13 \mathrm{k}^{5}-88440 \mathrm{k}^{3}-2036800 \mathrm{k}^{2}\right. \\
& +174852880 \mathrm{k}+5492560128)\left(2 \mathrm{k}^{5}-29480 \mathrm{k}^{3}+814720 \mathrm{k}^{2}+42414720 \mathrm{k}+511926272\right)=0 .
\end{aligned}
$$

Expandindo a igualdade acima teremos:

$$
\begin{gathered}
\mathrm{k}^{10}-44220 \mathrm{k}^{8}+407360 \mathrm{k}^{7}+588180720 \mathrm{k}^{6}-6377783808 \mathrm{k}^{5}-2743273620800 \mathrm{k}^{4} \\
+23766835860480 \mathrm{k}^{3}+3794883313628160 \mathrm{k}^{2}+200371311083520 \mathrm{k} \\
-707409499825373184=0
\end{gathered}
$$

Agora iremos analisar o termo independente da equação (19) de quinto grau e da equação (20) de décimo grau.

Notamos que na equação (19) o termo independente termina em 2, podemos então mudar as raízes dessa equação substituindo t por k-3 ou k+2 para que fique múltiplo de 5 . Vamos usar k-3:

$$
(k-3)^{5}-14740(k-3)^{3}+407360(k-3)^{2}+21207360(k-3)-511926272=0 \text {, }
$$

que resulta na equação:

$$
\mathrm{k}^{5}-15 \mathrm{k}^{4}-14650 \mathrm{k}^{3}+539750 \mathrm{k}^{2}+18365625 \mathrm{k}-571484375=0 \text {. }
$$


Agora faremos a mudança de variável na equação (20). Veja que a equação (20) termina em 4 .

Seguindo o mesmo raciocínio da equação de quinto grau, podemos substituir k na equação (20) por k-1.

$$
\begin{aligned}
& (\mathrm{k}-1)^{10}-44220(\mathrm{k}-1)^{8}+407360(\mathrm{k}-1)^{7}+588180720(\mathrm{k}-1)^{6}-6377783808(\mathrm{k}-1)^{5}- \\
& 2743273620800(\mathrm{k}-1)^{4}+23766835860480(\mathrm{k}-1)^{3}+3794883313628160(\mathrm{k}-1)^{2} \\
& +200371311083520(\mathrm{k}-1)-707409499825373184=0,
\end{aligned}
$$

Que resulta na equação:

$$
\begin{gathered}
\mathrm{k}^{10}-10 \mathrm{k}^{9}-44175 \mathrm{k}^{8}+761000 \mathrm{k}^{7}+584091250 \mathrm{k}^{6}-9895837500 \mathrm{k}^{5}-2702579343750 \mathrm{k}^{4}+ \\
34664405625000 \mathrm{k}^{3}+3707195755078125 \mathrm{k}^{2}-7307157128906250 \mathrm{k}- \\
703841490966796875=0 .
\end{gathered}
$$

Agora, para facilitar nosso raciocínio chamaremos o lado esquerdo da equação (22) de $\mathrm{g}(\mathrm{k})$ e a equação $(21)$ de $\mathrm{f}(\mathrm{k})$. Fazendo a divisão de $\mathrm{g}(\mathrm{k})$ por $\mathrm{f}(\mathrm{k})$, teremos como resto o polinômio:

$$
-9008 \mathrm{k}^{4}+4245920 \mathrm{k}^{3}-213340800 \mathrm{k}^{2}-4839268000 \mathrm{k}+287529550000=0 .
$$

Agora queremos encontrar uma solução para a equação:

$$
-9008 \mathrm{k}^{4}+4245920 \mathrm{k}^{3}-213340800 \mathrm{k}^{2}-4839268000 \mathrm{k}+287529550000=0 .
$$

Com o uso do método de Descartes para solucionar uma equação do quarto grau descobrimos duas soluções inteiras: 55 e - 35. As demais soluções são desnecessárias uma vez que não são inteiras.

Se substituirmos k por 55 e -35 na equação (20) teremos:

$$
\begin{gathered}
(55)^{5}-15(55)^{4}-14650(55)^{3}+539750(55)^{2}+18365625(55)-571484375=0 \\
0=0 .
\end{gathered}
$$

$\mathrm{E}$

$$
\begin{gathered}
(-35)^{5}-15(-35)^{4}-14650(-35)^{3}+539750(-35)^{2}+18365625(-35)-571484375=0 \\
0=0 .
\end{gathered}
$$

Concluímos assim que 55 e -35 são soluções da equação (21). Agora iremos ver como obter as soluções da equação (18).

Lembre-se que usamos uma mudança de variável, no caso, $\mathrm{k}-3$, para poder deixar todas as soluções inteiras da equação por números que sejam múltiplos de 5. 
Conforme mencionado na seção 2.1 usaremos o termo $\frac{-\mathrm{b}+\mathrm{t}}{5 \mathrm{a}}$ para encontrar a solução da equação (18).

Da equação (18) temos que $b=-112$ e t nada mais é do que uma solução da equação (19). Porém temos que subtrair -3 da incógnita t para poder achar a solução da equação (18) pelo fato de termos feito uma mudança de variável que culminasse na equação (21). Então, segue que:

$$
\frac{-b+t}{5 a}=\frac{-112+(55-3)}{5}=\frac{-112+52}{5}=-12
$$

Logo, -12 é uma das soluções da equação (18). De fato, se substituirmos x por -12 na equação (18) teremos:

$$
\begin{gathered}
(-12)^{5}+112(-12)^{4}+4428(-12)^{3}+76032(-12)^{2}+551232(-12)+1244160=0 \\
0=0 .
\end{gathered}
$$

Mas, como temos outra solução da equação (20), -35 no caso, podemos novamente usar o termo $\frac{-\mathrm{b}+\mathrm{t}}{5 \mathrm{a}}$ para encontrar outra solução para a equação (18). A solução neste caso é -30 .

\section{Conclusão}

Ao final do artigo podemos concluir que uma equação do quinto grau completa pode sim ser resolvida de maneira analítica, basta usar os procedimentos descritos aqui. Quando uma equação de quinto grau possui soluções que não sejam inteiras, não é possível resolvê-la com o exemplo proposto aqui. No entanto, através do método proposto aqui é possível encontrar soluções de uma equação de quinto grau quando a equação for possível de ser resolvida por radicais mesmo se suas raízes forem números irracionais.

\section{Referências}

ARTIN, E.: Galois Theory, Dover Publications, 1998.

GREEN, M. L. On the analytic solution of the equation of fifth degree. Compositio Mathematica, t. 37, n. 3, p. 233-241, 1978

STEWART, I. Galois theory. 3rd ed. Boca Raton: Chapman \& Hall/CRC, 2004. Disponível em: https://math.illinoisstate.edu/schebol/teaching/40714-files/Stewart-galois_theory.pdf. Acesso em: 03 dez. 2019.

ZOLADEK, H. The topological proof of Abel-Ruffini theorem. Topological Methods in Nonlinear Analysis, v. 16, p. 253-265, 2000. Disponível em: https://projecteuclid.org/download/pdf_1/euclid.tmna/ 1471875703. Acesso em: 03 dez. 2019. 